\title{
BMJ Open Risk of perinatal mortality in the first year of midwifery practice in New Zealand: analysis of a retrospective national cohort
}

\author{
Lynn C Sadler, ${ }^{1,2}$ Judith McAra-Couper, ${ }^{3}$ Deborah Pittam, ${ }^{4,5}$ Michelle R Wise, ${ }^{6}$ \\ John M D Thompson ${ }^{7}$
}

To cite: Sadler LC, McAraCouper J, Pittam D, et al. Risk of perinatal mortality in the first year of midwifery practice in New Zealand: analysis of a retrospective national cohort. BMJ Open 2018;8:e019026. doi:10.1136/ bmjopen-2017-019026

- Prepublication history for this paper is available online. To view these files, please visit the journal online (http://dx.doi. org/10.1136/bmjopen-2017019026).

Received 7 August 2017 Revised 15 February 2018 Accepted 19 February 2018

Check for updates

For numbered affiliations see end of article.

Correspondence to

Dr Lynn C Sadler;

lynns@adhb.govt.nz

\section{ABSTRACT}

Objectives To determine whether there was an increased risk of perinatal mortality among mothers booked for care with community lead maternity carer (LMC) midwives in their first compared with later years of practice.

Design Retrospective cohort study using linked national maternity, mortality and workforce data; adjusted analysis using logistic regression.

Setting New Zealand.

Participants Women under community LMC midwifery care birthing 2008-2014.

Main outcome measures Perinatal mortality (stillbirths and neonatal deaths of babies born from 20 weeks' gestation to the 27th day of postnatal life), excluding terminations and deaths associated with congenital abnormalities.

Results There were 2045 deaths among 344910 births booked with midwives. First year of practice midwives cared for women with higher risk of perinatal mortality, including Māori, Pacific, Indian, <20-year-old mothers, nullipara, smokers, women living in socioeconomic deprivation and with high body mass index, than midwives beyond first year of practice. There was a significant reduction in unadjusted odds of perinatal mortality among women under the care of midwives beyond the first year compared with those within the first year (OR 0.79, $95 \% \mathrm{Cl} 0.67$ to 0.93 ) but no significant reduction in risk remained after adjusting for known risk factors, (OR 0.89, $95 \% \mathrm{Cl} 0.74$ to 1.07$)$. There was a significant increase in the adjusted odds of perinatal mortality among midwives booking a caseload of 15 or fewer mothers per year (1.34, 1.01 to 1.78$)$ and 16 to $30(1.25,1.04$ to 1.50$)$ compared with midwives booking 51 to 80 .

Conclusions Findings suggest that the first year of midwifery practice is not associated with an increased risk of perinatal mortality but there is evidence that early career midwives are caring for higher-risk women. These findings suggest inequity of access for higher-risk women to experienced midwives and highlight an opportunity to improve support for vulnerable women and new midwives.

\section{INTRODUCTION}

In New Zealand (NZ), maternity care is an integrated primary, secondary and tertiary service provided by the public health system,
Strengths and limitations of this study

- The study used data, on a complete national cohort of births under midwifery care, obtained from three sources: the Ministry of Health database of births, the Perinatal and Maternal Mortality Review Committee database of all perinatal deaths and the Midwifery Council database of registered midwives, with the express purpose of undertaking the planned analysis.

- The data were linked using unique patient National Health Indices and midwifery practice numbers.

- The research group included appropriate statistical expertise and appropriate personnel with significant national knowledge of midwifery and obstetric practice.

- Some of the data the study used were originally collected for routine counting and funding purposes rather than for research.

- The observational nature of the study can lead to inherent biases, both known and unknown.

free of charge. ${ }^{1}$ Primary maternity continuity care is provided in the main by caseloading community-based midwives, known as lead maternity carers (LMCs), who are supported by a number of frameworks including national referral guidelines ${ }^{2}$ which enable midwives to work with obstetricians, paediatricians, general practitioners (GPs) and others.

In late 2015, a study was published by Lawton et al which reported an increased risk of perinatal mortality in NZ from 2005 to 2009 , for pregnancies cared for by community LMC midwives (previously known as self-employed midwives) within 1 year of Midwifery Council (MC) registration compared with midwives with 5-9 years of post-registration experience. ${ }^{3}$ However, the interpretation of the study findings was based on univariable analysis of observational data despite evidence in the data of increased risk among the women cared for by midwives in their first 
year of midwifery practice. The magnitude of the increase in risk of perinatal death was small Relative risk (RR 1.33 (1.02-1.73)), and there was no clear justification for the reference group of 5-9 years of practice in preference to more experienced midwives. The authors are not aware of studies from other countries investigating the impact of years of midwifery experience on perinatal mortality. A study from Israel, published in 2017, showed a decreased risk of third-degree and fourth-degree perineal tears with increasing years of midwifery experience. ${ }^{4}$

This study aimed to determine whether years of midwifery experience were associated with perinatal mortality among women booked for maternity care with community LMC midwives in NZ between 2005 and 2014, after controlling for potential confounding variables.

Specifically, the study aimed to reanalyse the 20052009 cohort of births cared for by midwives in the study by Lawton $e t a \hat{l}$ to determine whether the findings were valid given adjustments for potential confounders. If there was an increased risk of perinatal mortality among women under the care of community LMC midwives in their first year of midwifery practice from 2005 to 2009, then the study would analyse a later cohort of births from 2010 to 2014 using the same methodology. The study also aimed to examine whether midwifery caseload, nursing training prior to midwifery training or training overseas compared with in NZ had independent effects on perinatal mortality.

\section{METHODS}

A dataset of babies born during 2005-2009, and then a second set 2010-2014, were prepared by the NZ Ministry of Health by linking data from the National Maternity Collection (MAT), the National Minimum Dataset (NMDS), the National Mortality Collection (MORT), MC and the Perinatal and Maternal Mortality Review Committee (PMMRC).

The MAT is compiled from data collected by LMCs, the National Health Index (NHI) dataset (which collects demographic data) and hospital discharge data from the NMDS. The NMDS is the repository of International Classification of Diseases, Tenth Edition (ICD-10)-coded data from hospital visits of at least 3 hours (inpatient). The MC provided midwifery qualification and registration data, and the responses to surveys completed when midwives requested an annual practicing certificate, for the identified midwives. Survey data were linked to births in the same year as the year the survey was completed. The PMMRC dataset is a national dataset of all perinatal deaths ascertained via a network of local coordinators in District Health Boards (DHBs) and LMCs and is recognised as the gold standard for complete and accurate perinatal mortality data in NZ.

Data linkages between MAT, NMDS, MORT and PMMRC databases were made using baby and/or mother unique NHIs and linkages between the baby-mother clinical dataset and the MC data were made using LMC registration number and/or name. All NHI and LMC number and name data were removed before the data were provided to the investigators.

Each year, approximately 60000 babies are born in NZ, and 600-700 babies suffer perinatal death, defined as death of a baby born from 20 completed weeks gestation, either in utero (stillbirth) or during the first 27 days of life (neonatal death). ${ }^{5}$

Analyses were restricted to women/baby pairs with a community LMC midwife at booking (usually the first antenatal visit) by including only births where there was a record in the MAT that the LMC at booking was a midwife and where there was a record of the midwife in the MC database. The term 'community LMC midwife' is used throughout this manuscript to describe a midwife working independently, providing continuity of maternity care, responsible for antenatal, intrapartum and postpartum management of the woman and her baby, supported by the guidelines for consultation with obstetric and related medical services and under Section 88 of the Primary Maternity Services Notice 2007. ${ }^{26}$ The term 'booking' is used in this paper in place of the current term 'registration' for the antenatal visit where a woman engages a midwife to provide antenatal care to avoid confusion with the concept of midwifery registration with the MC. Terminations of pregnancy (TOP) and deaths with congenital abnormalities were excluded from the analysis as these were not believed to be associated with LMC care. TOPs were identified as such in the PMMRC dataset; congenital abnormalities were identified using the Perinatal Society of Australia and New Zealand (PSANZ) perinatal or neonatal death classification codes for congenital abnormality or ICD-10 Australian Modification codes for congenital abnormality as underlying cause of death where PSANZ codes were not available (ICD codes: Q00Q99, I424). ${ }^{7}$

The following variables were included in the analysis:

1. Maternal data: age, ethnicity (prioritised as per Ministry of Health guidelines), ${ }^{8}$ deprivation decile, parity, multiple pregnancy, smoking and body mass index (BMI) at booking with the LMC (BMI was calculated from height and weight with out of range heights $(<120 \mathrm{~cm}$ and $>200 \mathrm{~cm})$ and weights $(<40 \mathrm{~kg}$ and $>300 \mathrm{~kg}$ ) set to missing); hypertension (ICD 10 codes O100-O109, O11, O13, O140-O149, O150O159, O16), diabetes (ICD codes O240-O249), antepartum haemorrhage (ICD codes O200-O209, with at least one of O093-O095 or O460-O469; or O670O679) or placenta praevia (ICD codes O040-O049) which are coded at hospital discharge (including discharge after the birth admission).

2. Neonatal data: sex, year of birth/death and customised birthweight centile (derived from sex, birth weight, gestation, maternal ethnicity, parity, height and weight $)^{9}$ and categorised as small for gestational age $(<10$ th centile), appropriate for gestational age (10th - 90th centile) and large for gestational age (>90th centile). ${ }^{10}$ 
3. Midwifery data: LMC at booking, LMC at delivery, years from first midwifery registration, prior nursing qualification, country of training and annual midwifery caseload.

Deprivation decile and year of birth were included as ordinal variables in multivariable analyses. In univariable analyses, deprivation decile is presented as collapsed quintiles for ease of comprehension. Deprivation decile is an area-based measure of socioeconomic deprivation derived from data on internet access, income, housing, education, employment, marital status and access to a car, collected in the $2006 \mathrm{NZ}$ Census of dwellings and place of residence, measured from 1 (least deprived) to 10 (most deprived). ${ }^{11}$ All other variables were categorical, based on prior reported associations with perinatal mortality.

It is not clear whether a change of LMC during pregnancy or birth is a good indicator of a change in risk status as LMC may change for other reasons such as a woman's personal preference. More importantly, there is more often a change in clinical responsibility without a change in LMC. $^{12}$ Change in clinical responsibility from a community LMC midwife to an obstetrician is not captured in NZ datasets. In some instances of perinatal death, the person with clinical responsibility at birth may be more important than the LMC at booking but this is not captured in the data currently collected and so was not explored in the analysis.

Midwifery years of experience for NZ-trained midwives who registered with the MC after 1 December 2007 was calculated using multiples of 365 days following booking of the first woman with the new midwife LMC. NZ-trained midwives who registered with the MC prior to 1 December 2007 had their first year of midwifery practice counted from date of registration. For these midwives, their first year may have been shortened due to the delay between registration with the $\mathrm{MC}$ and starting to build a caseload, and for some there may have been no women birthed during the first year. However, most midwives working as LMCs during their first year of practice will have been accurately captured in the study period 2008-2014. For overseas-trained midwives, years of midwifery experience were counted from the date of first registration in their country of training. Years of midwifery experience were not calculated for midwives with unknown country of first registration (as provided by the MC) as there was no known date to be used as a starting date. Midwifery experience was analysed (a priori) in three ways (1) dichotomised as first year and beyond first year, (2) categorised as 1st, 2nd-5th, 6th-10th, 11th-20th, beyond 20th year and (3) in the form defined in the previous analysis (1st, 2nd, 3rd, 4th, 5th, 6th-10th, 11th-20th, beyond 20th year). ${ }^{3}$

A variable was derived for caseload per year of practice. Caseload was calculated over 1 year with the starting point of the year being the anniversary of the first date of booking of a woman with the LMC. This is an absolute count of births where the woman first booked with the index midwife in the year. No account was made in this measure of changes of LMC. At the end of each midwife's years during the study period, there is a truncated (or right censored) year. As there is potential for this to bias the measure, the caseload for the final year was counted backwards from the last booking with the index midwife. If a midwife contributes fewer than 365 days of bookings and the first and last of these dates is between 1 January 2008 and 1 March 2014, then no adjustment was made. If a midwife contributes fewer than 365 days of bookings and the first of these dates is after 1 March 2014, then an adjustment was made to annualise the caseload as this was assumed to be a midwife just starting practice in NZ. If a midwife contributes fewer than 365 days of bookings and the last booking date is before 1 January 2008, then adjustment was made as this was assumed to be a midwife just finishing practice in NZ. If there is only one booking with any midwife in the complete dataset, then no adjustment was made as the adjustment is too speculative (it would be 365 cases). There were 91 of these in the complete dataset. A generalised additive model (GAM) was fitted to assess the shape of the relationship of the continuous caseload variable to the binary perinatal mortality outcome. This showed a relatively linearly decreasing relationship up to approximately 80 deliveries per year and thereafter showed a slight increase in risk before increasing linearly again from approximately 120 deliveries per year. While the linear component of the GAM was significant ( $\mathrm{p}=0.0003$ ), it did not account for an adequate amount of deviance $(\mathrm{p}<0.0001)$. As such, based on the distribution of the data and midwifery advice, caseload was categorised in the following groups $(\leq 15,16-30$, $31-50,51-80$ and $>80 /$ year). Fifty-one to 80 was chosen as the referent category as it is the most common and associated with the lowest unadjusted perinatal mortality risk. Country of training and previous nursing training were obtained from the MC survey completed each year on application for an annual practising certificate.

PMMRC data were used for some variables to improve accuracy, but only where systematic differences that might create numerator-denominator bias were unlikely. Specifically, birth status (stillbirth, termination of pregnancy, neonatal death), gestational age, birth weight and plurality were obtained from the perinatal mortality dataset in preference to MAT data. In all other instances, MAT data were used for numerator and denominator.

\section{Statistical methods}

Statistical analysis was performed using STATA V.13 and SAS V.9.3.

Univariable statistics were estimated for associations between the midwifery experience variable and the outcome variable perinatal death, descriptive variables and all potential confounders and tested using a $\chi^{2}$ test. Univariable tests for trend were performed using an appropriate nominal variable to represent years of midwifery experience or caseload to determine if linear relationships existed with perinatal mortality rate.

Multivariable analyses adjusted for potential known confounders in the association between midwifery 
experience and outcomes, based on a priori hypotheses. ${ }^{3}$ The a priori variables included in the model represented maternal age, socioeconomic deprivation, BMI, ethnicity, smoking, parity, diabetes, hypertension, multiple pregnancy, antepartum haemorrhage, year of baby's birth, baby sex and customised birthweight centile. No variables were excluded from the final model. Missing data were not imputed. Unadjusted rates and crude and adjusted ORs with $95 \%$ CIs are presented with a nominal $p$ value $<0.05$ considered to denote statistical significance throughout.

The final multivariable model was used to perform sensitivity analyses including births from 23 weeks, births at term (from 37 weeks) and excluding births where there was a formal transfer of LMC in pregnancy to medical (GP or obstetrician) or DHB (secondary) care. Further models were also run including only socioeconomic deprivation, age, parity, ethnicity, with and without midwifery caseload.

There was no patient or consumer involvement in this study.

\section{RESULTS}

\section{Dataset of births}

It was not possible to reconstruct the dataset for the 20052009 analysis because the data were not able to distinguish women cared for by community LMC midwives from other categories of caregiver using either the variable in the MAT dataset or with MC data. A decision was made to abandon attempts to recreate the 2005-2009 dataset and to revise the initial plan. The final dataset used for this study included cases from 2008 to 2009, as it was possible from 2008 onwards to determine caregiver, combined with data from 2010 to 2014.

There were 442030 babies included in the dataset of births from 2008 to 2014, including live born and stillborn babies (figure 1). There were 4731 perinatal-related deaths from the PMMRC dataset. After the merge, there were 441914 babies, including 4586 matched PMMRC perinatal-related deaths (96.9\%) and 437328 surviving babies, along with 116 unmatched perinatal-related deaths from the MAT dataset. Seventy perinatal deaths from the PMMRC dataset did not merge because they had no valid NHI, and no match was found for 75 . The 116 deaths in the MAT dataset that did not merge with PMMRC deaths were retained on the assumption that these represented the unmatched PMMRC deaths.

\section{Dataset of midwives}

Of the original dataset of 442030 babies, 346121 (78.3\%) were first registered under community LMC midwifery care. Of these, $345922(99.9 \%)$ also had a midwifery number that matched with the MC database.

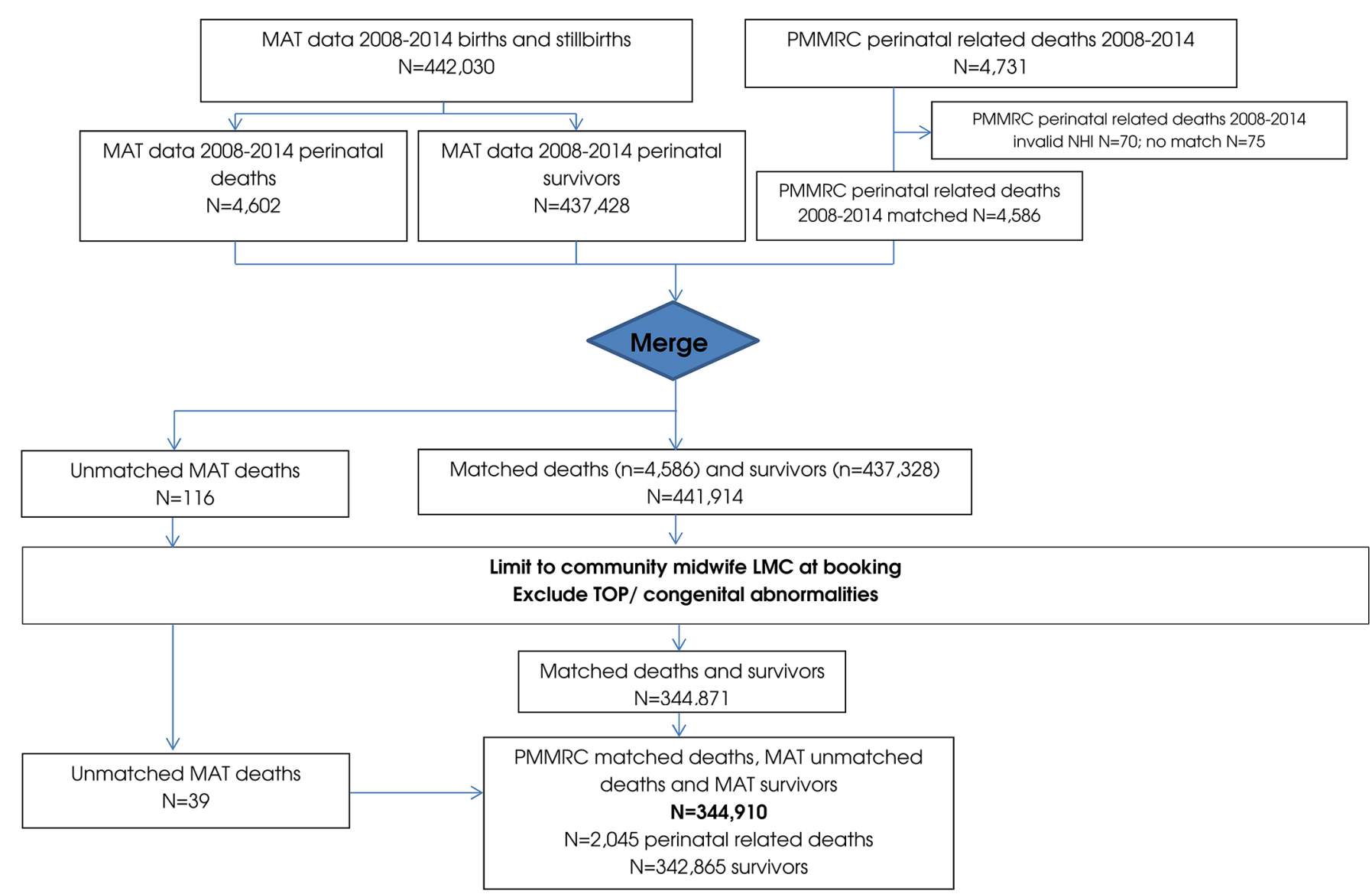

Figure 1 Compiling the dataset. LMC, lead maternity carer; MAT, National Maternity Collection; NHI, National Health Index; PMMRC, Perinatal and Maternal Mortality Review Committee; TOP, terminations of pregnancy. 
After limiting the dataset to women cared for by community LMC midwives, and excluding TOP and deaths with congenital abnormalities, there were 344871 babies in the merged set and 39 unmatched deaths. The unmatched deaths were included in the set to make a final dataset of 344910 births (figure 1).

Of the 2045 perinatal-related deaths included in the final dataset for analysis, 1433 were stillbirths and 612 neonatal deaths.

There were missing data for midwifery years of experience for 25425/344910 (7.4\%) of births. Data were also missing on previous nursing training (20364; 5.9\%) and country of training $(8450 ; 2.4 \%)$ in a subset of these. There was a change of LMC type from booking to birth for $5.2 \%$ of births, $4.8 \%$ to a DHB or other LMC and $0.4 \%$ to a private obstetrician or GP.

Among births in the period 2008-2014, where mothers first registered with a community LMC midwife, 19948 $(6.2 \%)$ were registered with a midwife in their first year of LMC practice. Of the remainder, 60894 (19.1\%), 66474 (20.8\%), $96855(30.3 \%)$ and $75314(23.6 \%)$ were registered with 2nd-5th, 6th-10th, 11th-20th and midwives beyond 20 years of practice, respectively (table 1 ).

\section{Midwifery experience and perinatal deaths}

First year of practice midwives were more likely to care for Māori, Pacific and Indian mothers, young mothers, women living in areas of higher socioeconomic deprivation, nullipara, smokers and women with high BMI compared with midwives beyond first year (table 1). Midwives in their first year were also more likely to care for babies who went on to be born small for gestational age, less likely to care for mothers with multiple pregnancies and more likely to care for mothers with hypertension, type 2 diabetes and antepartum haemorrhage compared with midwives beyond first year (table 2).

Unadjusted analysis shows a statistically significant reduction in odds of perinatal mortality among women under the care of midwives beyond first year of practice compared with those in first year of practice (perinatal mortality rates $5.85 / 1000$ births and 7.42/1000 births, respectively) (OR 0.79 (0.67 to 0.93)) (table 3). After adjusting for known potential confounders, the reduction in risk was no longer statistically significant (OR 0.89 (0.74 to 1.07$)$ ). Inclusion of only socioeconomic deprivation, age, parity and ethnicity in the multivariable model accounted for most of the changes in OR (OR 0.87 (0.73 to 1.03)). The further addition of midwifery caseload results in a similar adjustment in OR as the full model (OR 0.89 (0.74 to 1.06$)$ ).

The adjusted multivariable findings were robust after performing sensitivity analyses including only births from 23 weeks (OR 0.84 (0.68 to 1.03$)$ ), at term (0.89 (0.64 to 1.24)) and after excluding transfer of LMC to medical or DHB care from the final multivariable model $(0.87$ (0.72 to 1.05$)$ ).

Considering the length of midwifery experience in more detail found a statistically significant effect in univariable analysis when categorised into five groups $\left(\chi^{2}=13.04, p=0.011\right)$, but no significant test for linear trend was detected $\left(\chi^{2}=1.15, \mathrm{p}=0.28\right)$. There was a significantly lower perinatal mortality, after adjusting for known potential confounders, among women under the care of 2nd-year to 5th-year midwives (OR 0.81 (0.66 to 0.99$)$ ) compared with 1st year of practice midwives. The odds for all other more experienced midwives compared with 1st year of practice midwives were not significant and close to $1.00(0.97,0.95$ and 0.92 for midwives in their 6 th-10th, 11th-20th and beyond 20 years of midwifery experience). In other words, if 2nd to 5th years of experience are set as the referent group, then 1st year of practice midwives have increased odds of perinatal mortality (OR 1.24 (1.01 to 1.52$)$ ), with similar odds to 6th-10th year (OR 1.20 (1.03 to 1.40$)$ ), 11th-20th year (OR 1.18 (1.00 to 1.39$)$ ) and greater than 20 years' experience (OR 1.14 (0.94 to 1.39)).

Using the categories of experience in the previous study, ${ }^{2}$ there was no difference in odds of perinatal mortality for 6th-10th year compared with 1st-year midwives (OR 0.97 (0.79 to 1.18$)$ ). The only year where there was a significant reduction in odds was for midwives in their 5 th year of practice $(0.70$ (0.52 to 0.96$))$.

\section{Midwifery caseload}

There was a significant association between midwifery caseload and perinatal mortality in univariable analysis $\left(\chi^{2}=10.20, p=0.037\right)$, and a test for linear trend also resulted in a statistically significant result $\left(\chi^{2}=6.73\right.$, $\mathrm{p}=0.012$ ). In multivariable analysis, the adjusted odds of perinatal mortality remained stable and relatively unchanged from the univariable associations suggesting little confounding in relation to this association. The adjusted odds of perinatal mortality were significantly higher among midwives registering a caseload of 15 or fewer women per year (1.34 (1.01 to 1.78$)$ ) and 16-30 women per year (1.25 (1.04 to 1.50)) compared with midwives registering $51-80$ women per year. There was no significant difference between midwives with caseloads of $31-50$, or over 80 compared with midwives registering 51-80 women per year (table 3 ).

\section{Other midwifery factors}

There was no significant association between country of training or type of training (nurse-midwifery or directentry midwifery) and perinatal mortality in crude or adjusted analyses (table 3 ).

\section{DISCUSSION}

First year of practice community LMC midwives cared for higher risk women than midwives in later years of their careers. After adjusting for this and other contributory factors, the risk of perinatal mortality (stillbirth or neonatal death) for women under the care of first year of practice midwives was not significantly higher than for women cared for by midwives beyond their first year. Years 


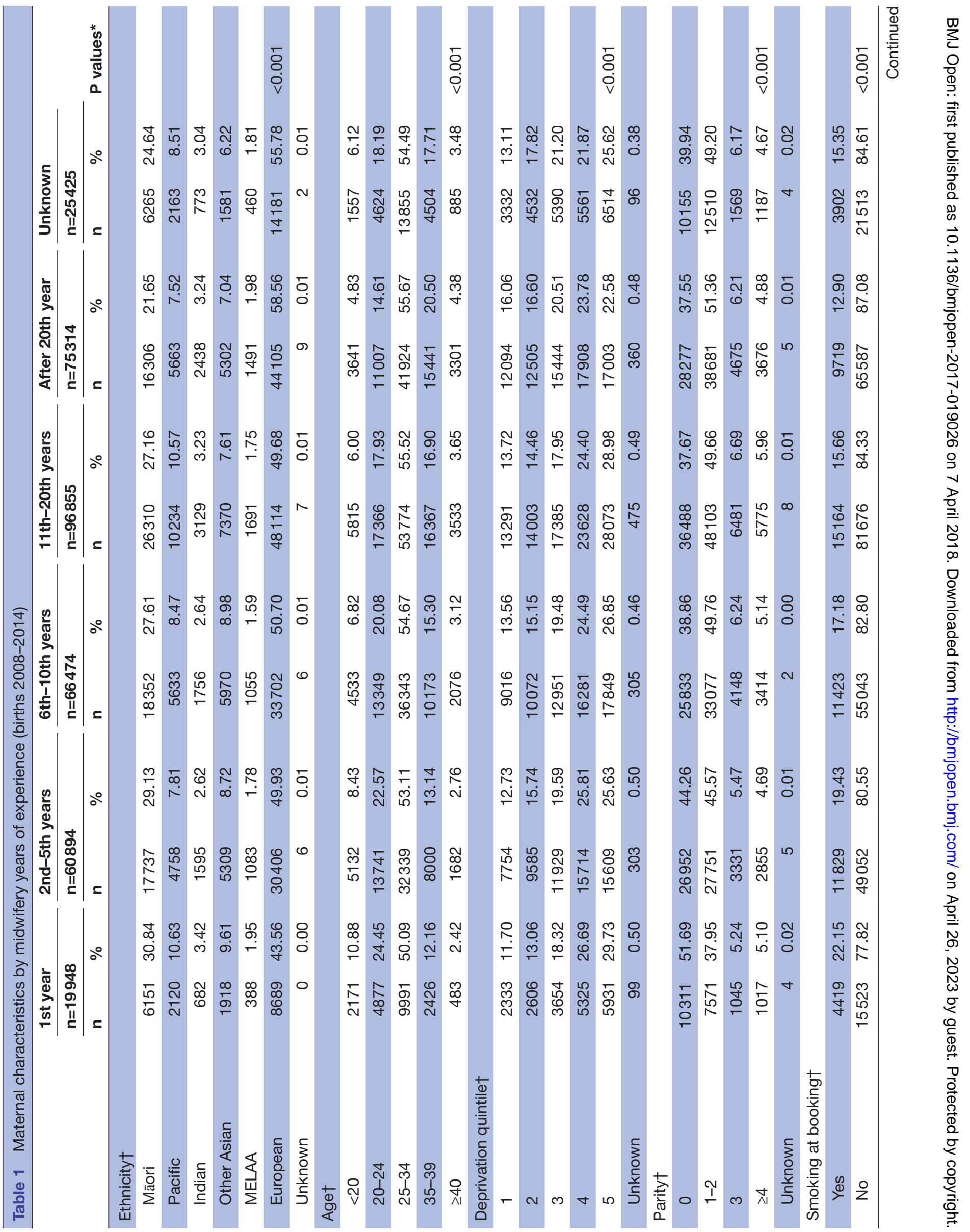




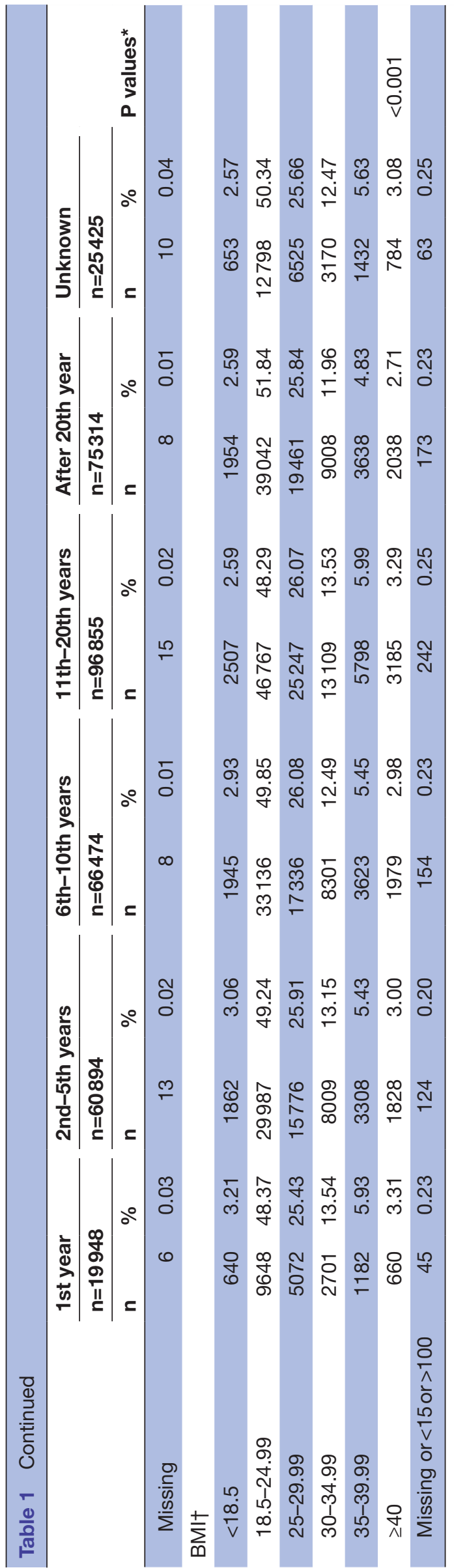

of midwifery experience were categorised into subgroups in an attempt to replicate the analysis in the previous paper from NZ. ${ }^{3}$ This showed that first year of practice midwives had the same odds of perinatal mortality as midwives with five or more years of midwifery experience. Further, first year of practice midwives had the same odds of perinatal mortality as all midwives with the exception of midwives in their fifth year of practice. There was a protective effect in women cared for by midwives in their fifth year, but there is no apparent plausible reason for this reduced risk, and we consider it to be a chance finding possibly due to residual confounding. Based on current findings, there seemed no reason to identify an issue for midwives in their first year, and it seemed highly likely that years of experience did not relate to perinatal mortality. In addition, country of midwifery training and type of entry to midwifery training were not associated with perinatal death. However, carrying a caseload of fewer than 30 women per year was found to be associated with higher risk of perinatal mortality compared with a caseload of 51-80 women per year.

The main strength of this study was that it used data obtained directly from the Ministry of Health, the PMMRC and the MC, with the express purpose of undertaking the planned analysis. Despite the complexity of the concepts and limitations of the data, we paid considerable attention to detail in the methodology. Midwifery experience and caseload were represented as accurately as possible. The main limitation was that much of the data the study used were originally collected for routine counting and funding purposes rather than for research. Observational studies carry inherent risk of bias. As shown by the change in ORs with adjustment for the considered, identified and measured confounders, the predictor of interest (years of midwifery experience) was strongly associated with other predictors of perinatal mortality (such as antenatal risk factors). It is possible that residual confounding remains but this would be expected to reduce the estimated risk of first year of practice on perinatal mortality. Some risk factors for stillbirth, such as previous stillbirth, were not included in the analysis because they were not available in the dataset.

The study by Lawton et al published in 2016 caused considerable concern in the maternity workforce and in the wider community in NZ. ${ }^{3}$ Specifically, it eroded some of the community's trust in the unique midwifery model of care in place in NZ since 1990. In retrospect, Lawton's study presented univariable results only, despite providing evidence of potential confounding, and used data which were not fit for the purpose, hence the study could not be replicated. In the current study, when categorising years of midwifery experience as per Lawton $e t a l$, first year of practice midwives did not have significantly higher odds of perinatal mortality. It is possible that there was a concern during the previous study period that is no longer relevant; however, it is more likely that years of experience do not relate to perinatal mortality. The current study is important internationally as NZ has a 


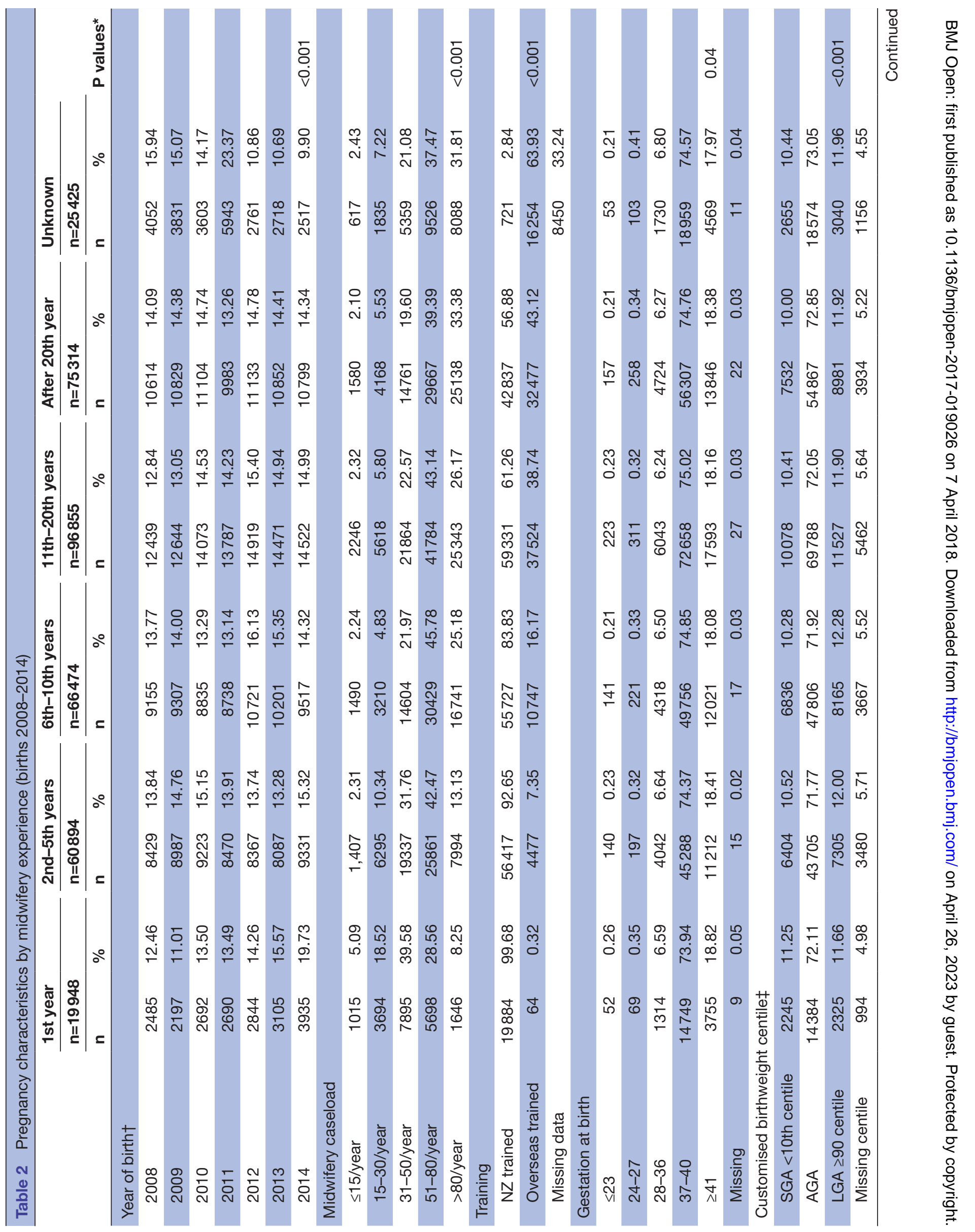




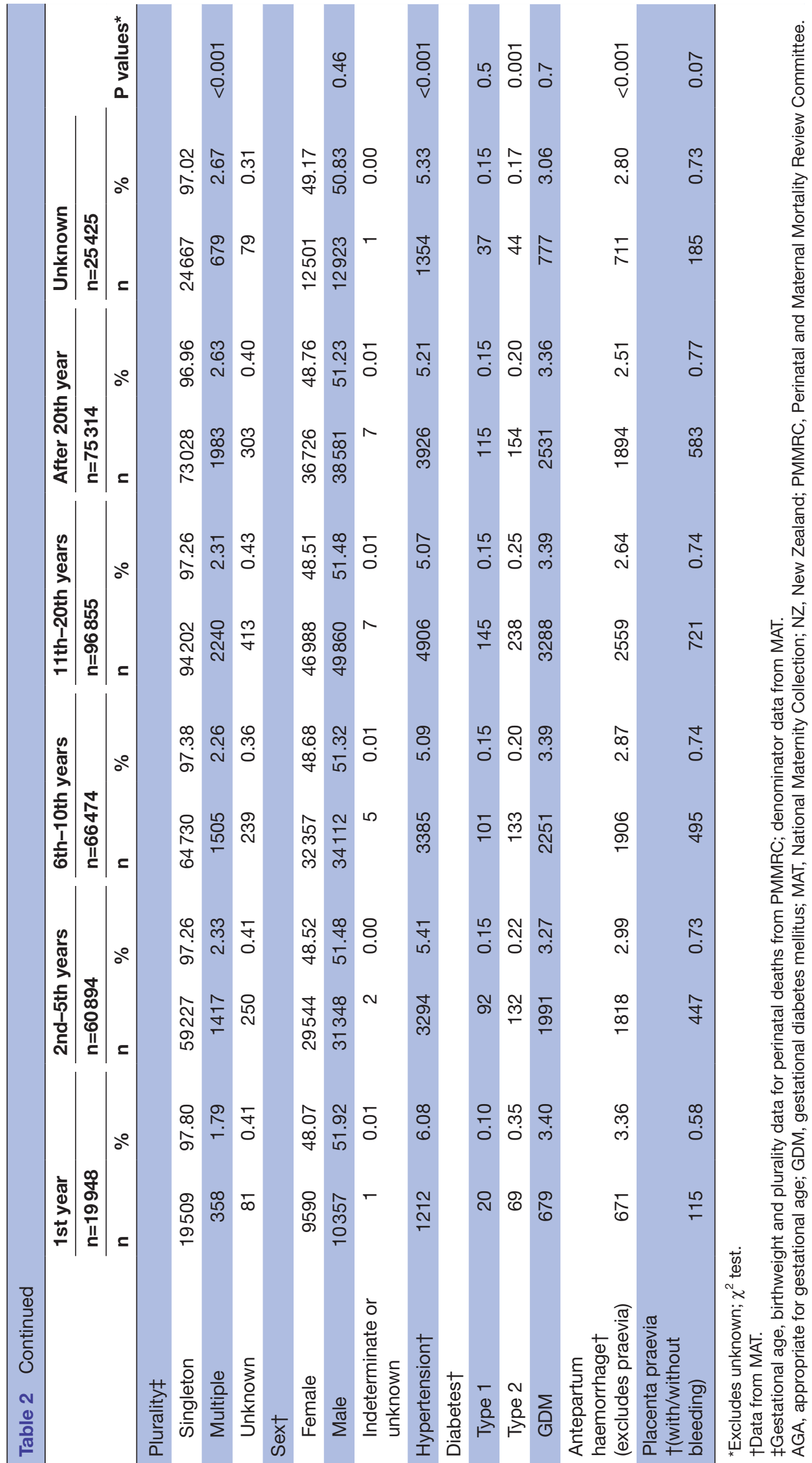




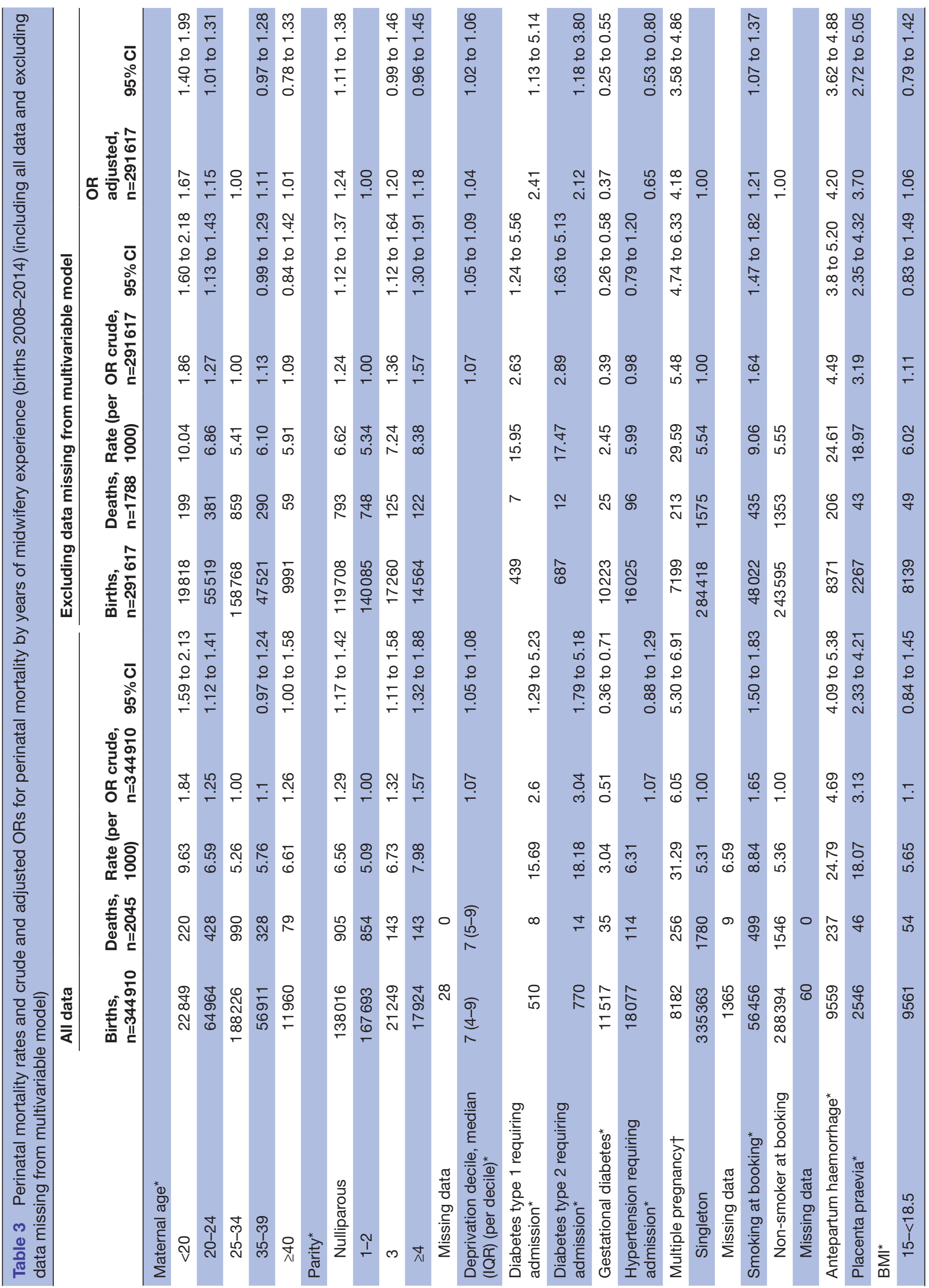




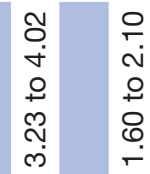

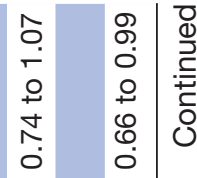

कृष

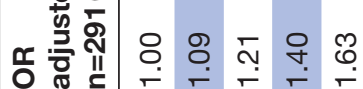

Øூ

8 웅 8

๕̊

$\begin{array}{lll}\infty & \infty \\ \infty & \infty \\ 0 & \infty \\ 0 & 0 \\ 0\end{array}$

이 우 오 두

ๆ เ

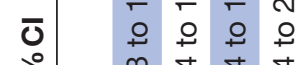

广

ํำ

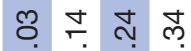

는 둔 운

ธ.

న 8

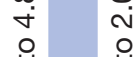

๙ั กิ

กุ ฮั

요앙

ลัด

ํํㄹ 든

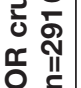

움

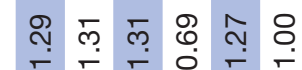

ஜ용

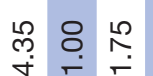

$\begin{array}{lll} & \infty & \\ \infty & 0 \\ 0 & 0 & 0 \\ 0 & 0 & 0 \\ 0\end{array}$

ळ

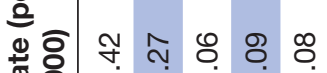

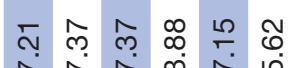

$\stackrel{0}{4} \frac{1}{9}$

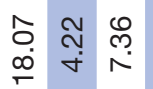

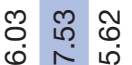

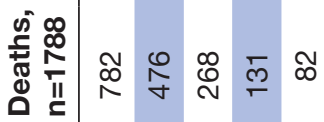

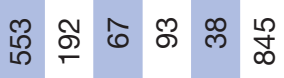

임 $\frac{\infty}{\infty}$

ํํ요 ๙

过 ำ

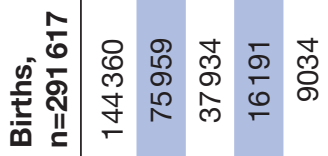

N

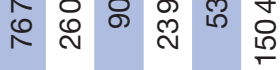

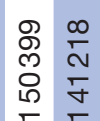

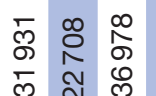

요 $\infty$

N

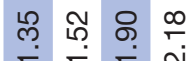

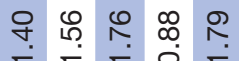

- 다웅

‘் ‘́

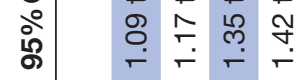

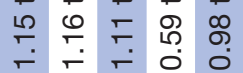

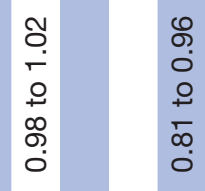

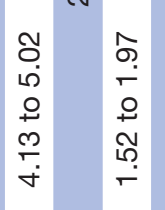

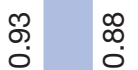

$\circ$

윟웅

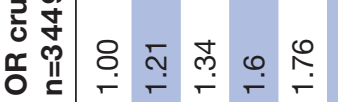

స়

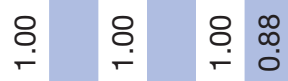

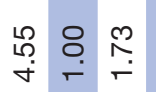

仓ิ

$\stackrel{\circ}{\varrho}$

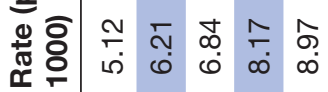

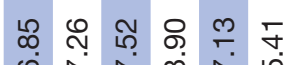

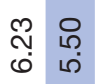

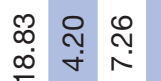

œ

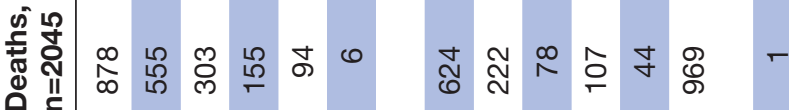

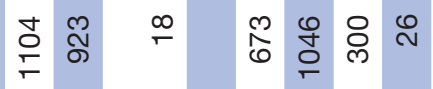

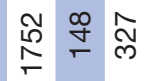

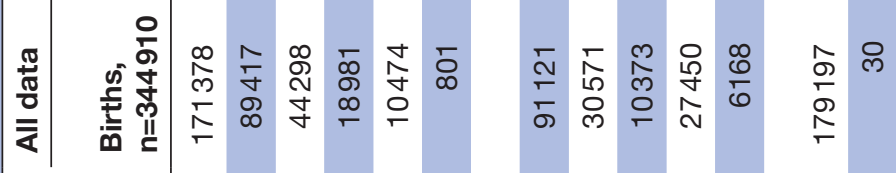

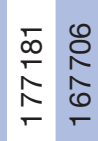

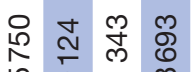

岗

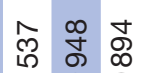

令

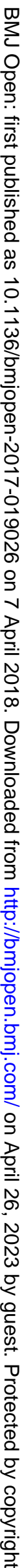

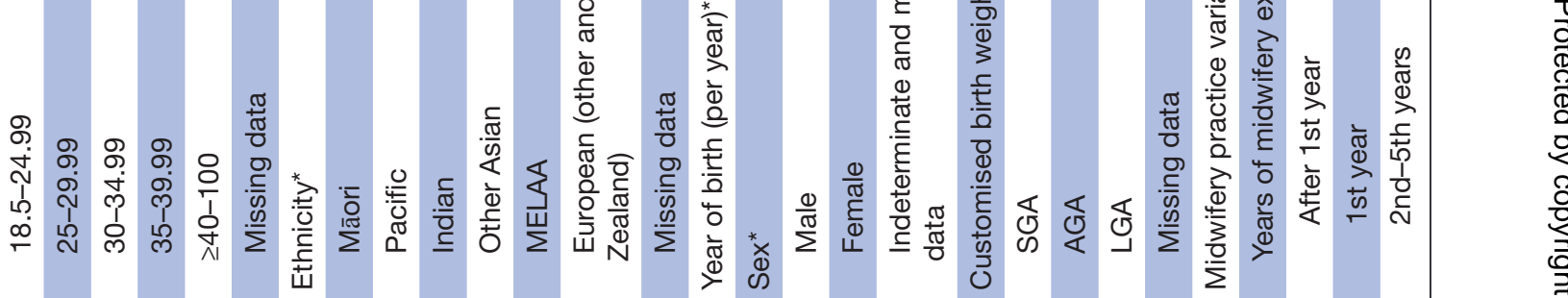

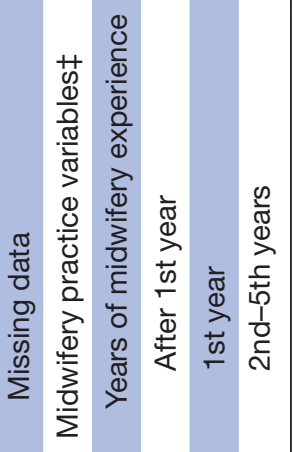




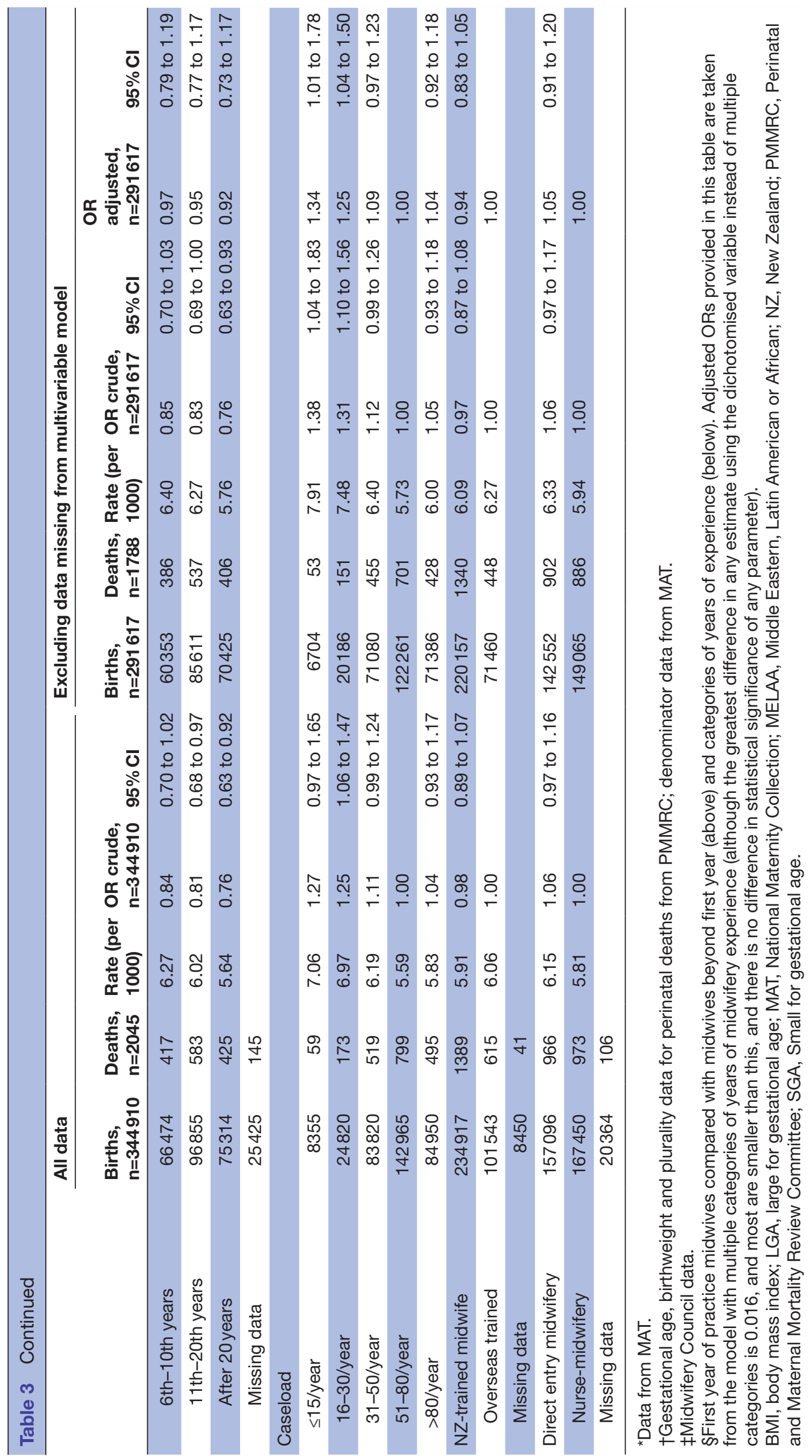


unique maternity model of care supported by integrated primary, secondary and tertiary services and is a model for the development of midwifery models worldwide. Community LMC midwives provided primary maternity care to $85.2 \%$ of the birthing population in NZ in $2014 .{ }^{13}$

While the current study is reassuring with regard to the risk of perinatal mortality related to years of midwifery experience, it also raises two important issues. First, it indicates a higher level of clinical risk among the women who book with midwives in their first year of practice. This may suggest inequitable access to experienced LMC midwives. It is possible this occurs because of the difficulties of building a caseload in the first year when the midwife does not have a reputation or repeat clients (who have become multiparous). More research is needed to understand this complex relationship, and the profession needs to consider options for further support for midwives in their first year who are caring for higher risk women. Second, it suggests that caseload may be important among midwifery LMCs. It remains to be determined why low caseload was found to be associated with perinatal mortality.

\section{Author affiliations}

${ }^{1}$ Women's Health, Auckland District Health Board, Auckland, New Zealand ${ }^{2}$ Department of Epidemiology and Biostatistics, Faculty of Medical and Health Science, University of Auckland, Auckland, New Zealand

${ }^{3}$ Midwifery Department, Auckland University of Technology, Auckland, New Zealand ${ }^{4}$ Maternity Services, Northland District Health Board, Whangarei, New Zealand

${ }^{5}$ New Zealand College of Midwives, Christchurch, New Zealand

${ }^{6}$ Department of Obstetrics and Gynaecology, Faculty of Medical and Health Sciences, University of Auckland, Auckland, New Zealand

${ }^{7}$ Departments of Paediatrics, Child and Youth Health, and Obstetrics and Gynaecology, Faculty of Medical and Health Science, University of Auckland, Auckland, New Zealand

Contributors LCS and JMDT conceived the protocol for the study and performed the analysis. The first author wrote the first draft of the paper. JMA-C, DP and MRW provided advice on the protocol, the analysis and interpretation of the findings. All authors were involved in editing the paper. LCS is the guarantor for the study. The first and last authors had access to the data throughout the study while the other authors did not. The lead author affirms that the manuscript is an honest, accurate and transparent account of the study being reported; that no important aspects of the study have been omitted and that any discrepancies from the study as planned have been explained. The authors requested that the study findings be submitted for publication and were responsible for preparation of the manuscript.

Funding The first and last authors of this study were supported by the New Zealand Ministry of Health and the Accident Compensation Commission (ACC) of New Zealand.

Disclaimer The funder (NZ Ministry of Health) requested a replication of the original study, but did not contribute to the study design, analytical plan, analysis or interpretation of the data. The authors approached the funders when it was clear that the initial study analysis could not be completed and proposed a change of the timeframe to 2008-2014. The sponsors/funders have not reviewed the paper prior to submission. All decisions were independent of the funders.

Competing interests JMCA-C is paid as the Chair of the Midwifery Council of NZ and DP is paid as the President of the NZ College of Midwives
Patient consent Not required.

Ethics approval New Zealand Northern A Health and Disability Ethics Committee (16/NTA/1).

Provenance and peer review Not commissioned; externally peer reviewed.

Data sharing statement The data for this study are not available for sharing; however, a similar dataset could be obtained from the New Zealand Ministry of Health, the New Zealand Midwifery Council and the PMMRC upon provision of usual ethical applications and consents.

Open Access This is an Open Access article distributed in accordance with the Creative Commons Attribution Non Commercial (CC BY-NC 4.0) license, which permits others to distribute, remix, adapt, build upon this work non-commercially, and license their derivative works on different terms, provided the original work is properly cited and the use is non-commercial. See: http://creativecommons.org/ licenses/by-nc/4.0/

(c) Article author(s) (or their employer(s) unless otherwise stated in the text of the article) 2018. All rights reserved. No commercial use is permitted unless otherwise expressly granted.

\section{REFERENCES}

1. Gilkison A, Pairman S, McAra-Couper J, et al. Midwifery education in New Zealand: Education, practice and autonomy. Midwifery 2016;33:31-3.

2. Ministry of Health. Guidelines for consultation with obstetric and related medical services (Referral Guidelines). Wellington: Ministry of Health, 2012. http://www.health.govt.nz/system/files/documents/ publications/referral-glines-jan12.pdf (accessed 22 Jun 2017).

3. Lawton B, Filoche S, Geller SE, et al. A retrospective cohort study of the association between midwifery experience and perinatal mortality. Int J Gynaecol Obstet 2016;132:94-9.

4. Mizrachi $Y$, Leytes $S$, Levy M, et al. Does midwife experience affect the rate of severe perineal tears? Birth 2017;44:161-6.

5. PMMRC. Eleventh annual report of the perinatal and maternal mortality review committee: reporting mortality 2015. Wellington: Health Quality \& Safety Commission, 2017. https://www.hqsc.govt. nz/assets/PMMRC/Publications/2017_PMMRC_Eleventh_Annual Report.pdf (accessed 22 Jun 2017).

6. New Zealand Gazette. Maternity Services notice pursuant to Section 88 of the New Zealand Public Health and Disability Act 2000. 2007:41 http://www.health.govt.nz/publication/section-88-primarymaternity-services-notice-2007 (accessed 22 Jun 2017).

7. Flenady V, King J, Charles A, et al; Perinatal society of australia and new zealand clinical practice guideline for perinatal mortality. Version 2.2. Section 7: perinatal mortality classifications; appendix 1. Second Edition, 2009. https://psanz.com.au/assets/Uploads/Section-7Version-2.2-April-2009.pdf (accessed 22 Jun 2017).

8. Ministry of Health. Ethnicity data protocols for the health and disability sector. Wellington: Ministry of Health, 2004. http://www. health.govt.nz/system/files/documents/publications/ethnicityda taprotocols.pdf (accessed 26 Jul 2017).

9. Gardosi J, Francis A. Customised weight centile calculator: GROW v6.7.8, Gestation Network, 2016. http://www.gestation.net

10. Anderson NH, Sadler LC, Stewart AW, et al. Maternal and pathological pregnancy characteristics in customised birthweight centiles and identification of at-risk small-for-gestational-age infants: a retrospective cohort study. BJOG 2012;119:848-56.

11. Salmond C, Crampton P, Atkinson J. NZDep2006 index of deprivation. Wellington: Department of Public Health, University of Otago, 2007. http://www.otago.ac.nz/wellington/otago020337.pdf (accessed 26 Jul 2017).

12. Skinner JP, Foureur M. Consultation, referral, and collaboration between midwives and obstetricians: lessons from New Zealand. $J$ Midwifery Womens Health 2010;55:28-37.

13. Ministry of Health. Report on maternity 2014. Wellington: Ministry of Health, 2015. http://www.health.govt.nz/system/files/documents/ publications/report-on-maternity-2014-dec15.pdf (Accessed 22 June 2017). 